\title{
Polyp Tip
}

National Cancer Institute

\section{Source}

National Cancer Institute. Polyp Tip. NCI Thesaurus. Code C83474.

The distal end of a polyp. 